\title{
QUADRUPOLE MOMENTS AND E2 TRANSITIONS IN THE O(6) LIMIT OF THE IBM
}

\author{
C.E. ALONSO, M. LOZANO \\ Departamento de Fisica Atomica y Nuclear, Universidad de Sevilla, E-41080 Sevilla, Spain
}

\section{C.H. DASSO}

The Niels Bohr Institute, University of Copenhagen, DK-2100 Copenhagen $\emptyset$, Denmark

and

\author{
A. VITTURI \\ Dipartimento di Fisica and INFN, I-38050 Povo, Trento, Italy
}

Received 3 March 1988; revised manuscript received 27 June 1988

\begin{abstract}
Quadrupole moments and E2 transitions in the $\mathrm{O}(6)$ limit of the interacting boson model are studied within a formalism based on the intrinsic frame. We show that the values of these quantities for both ground-and $\beta$-bands can be directly obtained, to higher order in $N$, by suitably projecting the corresponding matrix elements associated with the $\gamma$-dependent intrinsic states.
\end{abstract}

Within the geometrical model a particular situation, pointed out many years ago by Wilets and Jean [1], arises when the energy surfaces in the $\beta \gamma$-plane show a definite minimum for an equilibrium value $\beta_{0}$, but are otherwise independent of $\gamma$. This specific feature leads to a sequence of excited states and to values of quadrupole matrix elements different from those characterizing the rotational and vibrational spectra.

In the description of nuclear spectra given by the interacting boson model (IBM) a situation similar to that of $\gamma$-instability arises in the $O(6)$ limit [2], which displays energy spectra similar to those predicted by the Wilets-Jean model [3]. Concerning the quadrupole transitions, a crucial point in the IBM is the choice of the form of the associated operator

$\hat{Q}_{\mu}=\left[s^{\dagger} d\right]_{2 \mu}+\left[d^{\dagger} \tilde{s}\right]_{2 \mu}+\chi\left[d^{\dagger} d\right]_{2 \mu}$.

Its form has been particularly debated in connection with this limit. If one requires the quadrupole operator to be expressed in terms of the generators of the group, the $d^{\dagger} d$ term in (1) must, in the $\mathrm{O}(6)$ case, be excluded. This action in turn leads to the formulation of specific selection rules, such as no-crossover transition between the ground state and the second $2^{+}$ state in the spectrum and a vanishing quadrupole moment for all levels. It has been argued [4] that the failure of these selection rules detected in experiments should not be viewed as a breaking of the $O(6)$ symmetry, but rather as an indication for the need of retaining the $d^{\dagger} d$ term. Expressions for quadrupole moments and $B(\mathrm{E} 2)$-values in the $\mathrm{O}(6)$ limit have been given in ref. [4] for the general form (1) of the operator.

In this letter we show that the values of quadrupole moments and electromagnetic transition rates can be directly obtained exploiting the intrinsic state description of the IBM [5]. The selection rules and the interplay of the different terms of the quadrupole operator acquire in this approach a simple geometrical interpretation. Within this framework all the members of the ground-state band are viewed as originating from the intrinsic state

$\left|\Psi_{\mathrm{g}}\right\rangle=(1 / \sqrt{N !})\left(\gamma^{\dagger}\right)^{N}|0\rangle$

a condensate of the basic boson 


$$
\begin{aligned}
\gamma^{\dagger} & =\frac{1}{\left(1+\beta^{2}\right)^{1 / 2}}\left[s^{\dagger}+\beta \cos \gamma d_{0}^{\dagger}\right. \\
& \left.+(1 / \sqrt{2}) \beta \sin \gamma\left(d_{2}^{\dagger}+d_{-2}^{\dagger}\right)\right],
\end{aligned}
$$

which depends on the shape-like parameters $\beta$ and $\gamma$. These parameters are to be determined by minimizing the expectation value of the sd-boson hamiltonian for the particular case under consideration. In the case of the $O(6)$ hamiltonian the energy values become independent of $\gamma$ and show a minimum for $\beta=1$. The $O(6)$ ground band is therefore viewed as originating from a condensate of $\gamma$-dependent bosons

$$
\begin{aligned}
\gamma^{\dagger} & =(1 / \sqrt{2})\left[s^{\dagger}+\cos \gamma d_{0}^{\dagger}\right. \\
& \left.+(1 / \sqrt{2}) \sin \gamma\left(d_{2}^{\dagger}+d_{-2}^{\dagger}\right)\right] .
\end{aligned}
$$

In the familiar case of quadrupole-deformed objects the kernels connecting the intrinsic and laboratory frames are given by $D$-functions. In our case, where the intrinsic system is $\gamma$-dependent, this operation corresponds to a generalized rotation in five dimensions and the appropriate kernels are provided by the functions $\Phi_{\lambda, I, M}\left(\gamma, \theta_{i}\right)$, eigenfunctions of the free Bohr hamiltonian

$$
\begin{gathered}
\left(-\frac{1}{\sin 3 \gamma} \frac{\partial}{\partial \gamma} \sin 3 \gamma \frac{\partial}{\partial \gamma}+\frac{1}{4} \sum_{k=1}^{3} \frac{Q_{k}^{2}}{\sin ^{2}\left(\gamma-\frac{2}{3} \pi k\right)}\right) \\
\quad \times \Phi_{\lambda, I, M}\left(\gamma, \theta_{i}\right)=\lambda(\lambda+3) \Phi_{\lambda_{*}, M, M}\left(\gamma, \theta_{i}\right),
\end{gathered}
$$

and characterized by the quantum number $\lambda, I$ and $M$. Note that the quantum number $\lambda$, originally used in refs. $[1,6]$, corresponds to the quantum number $\tau$ of the $O(6)$ terminology. For details on the resulting spectra and on the $\Phi$ functions, we refer to refs. $[1,6]$. In the present context the only relevant functions are those with zero component of the angular momentum. To simplify the notation we shall henceforth drop from the labels in $\Phi$ the index corresponding to $M=0$. We quote here the explicit form of the functions associated with the lowest states, which depend only on the first two Euler angles $\theta$ and $\phi$,

$$
\begin{gathered}
\Phi_{1.2}(\gamma, \theta, \phi)=(1 / \sqrt{8 \pi})\left\{\cos \gamma Y_{20}(\theta, 0)\right. \\
\left.+(1 / \sqrt{2}) \sin \gamma\left[Y_{22}(\theta, \phi)+Y_{2-2}(\theta, \phi)\right]\right\}, \\
\Phi_{2.2}(\gamma, \theta, \phi)=(1 / \sqrt{8 \pi})\left\{\cos 2 \gamma Y_{20}(\theta, 0)\right. \\
\left.-(1 / \sqrt{2}) \sin 2 \gamma\left[Y_{22}(\theta, \phi)+Y_{2-2}(\theta, \phi)\right]\right\},
\end{gathered}
$$

and

$$
\begin{aligned}
& \Phi_{2,4}(\gamma, \theta, \phi)=(1 / 2 \sqrt{72 \pi}) \\
& \quad \times\left\{\left(6 \cos ^{2} \gamma+\sin ^{2} \gamma\right) Y_{40}(\theta, 0)\right. \\
& +\sqrt{\frac{15}{2}} \sin 2 \gamma\left[Y_{42}(\theta, \phi)+Y_{4-2}(\theta, \phi)\right] \\
& \left.+\sqrt{\frac{35}{2}} \sin ^{2} \gamma\left[Y_{44}(\theta, \phi)+Y_{4-4}(\theta, \phi)\right]\right\} .
\end{aligned}
$$

For large deformations, in correspondence to each intrinsic state one has in the laboratory frame a bandlike structure characterized by a sequence of levels whose angular momenta and energy spacing are governed by eq. (5). As an example all the members of the ground-state band are associated with the wavefunctions

$$
|\lambda, I, M\rangle_{\mathrm{g}}=\Phi_{\lambda, I, M}\left(\lambda, \theta_{i}\right)\left|\Psi_{\mathrm{g}}\right\rangle,
$$

where $\left|\Psi_{\mathrm{g}}\right\rangle$ is the intrinsic state defined in (2). This simple form holds, in the IBM approach, in the limit of large number of particles. As a consequence one can expect to reproduce energies and transition rates only in lowest order in $(1 / N)$. Within this scheme all matrix elements involve an integration over the variables $\gamma$ and the Euler angles plus the matrix elements in the intrinsic frame. In particular, matrix elements of the quadrupole operators between different states associated to the same intrinsic state can all be expressed in terms of the corresponding matrix elements in the $\gamma-\theta_{i}$ variables of a $\gamma-\theta_{i}$ dependent intrinsic quadrupole moment. One has in fact,

$$
\begin{aligned}
\mathrm{g} & \left\langle\lambda, I, 0\left|\hat{Q}_{0}^{\mathrm{lab}}\right| \lambda^{\prime}, I^{\prime}, 0\right\rangle_{\mathrm{g}} \\
& =\left\langle\Phi_{\lambda, I}\left|Q_{\mathrm{gg}}^{\mathrm{intr}}(\gamma, \theta, \phi)\right| \Phi_{\lambda^{\prime}, I^{\prime}}\right\rangle,
\end{aligned}
$$

where

$$
\begin{aligned}
& Q_{\mathrm{gg}}^{\mathrm{intr}}(\gamma, \theta, \phi)=\left\langle\Psi_{\mathrm{g}}\left|\hat{Q}_{0}^{\mathrm{lab}}\right| \Psi_{\mathrm{g}}\right\rangle \\
& \quad=\left\langle\Psi_{\mathrm{g}}\left|\sum_{\mu} \sqrt{\frac{4}{5} \pi}(-1)^{\mu} \hat{Q}_{\mu} Y_{2, \mu}(\theta, \phi)\right| \Psi_{\mathrm{g}}\right\rangle,
\end{aligned}
$$

which depends on $\gamma$ via the $\gamma$-dependence of the intrinsic state. The quantity (11) can be evaluated from eqs. (1) and (2), leading to

$$
\begin{aligned}
& Q_{\mathrm{gg}}^{\text {intx }}(\gamma, \theta, \phi)=4 \pi N \sqrt{\frac{2}{5}}\left[\Phi_{1,2}(\gamma, \theta, \phi)\right. \\
& \left.-(\chi / \sqrt{14}) \Phi_{2,2}(\gamma, \theta, \phi)\right] .
\end{aligned}
$$

We remark that it has been possible to reconstruct in (12) exactly the two functions $\Phi_{1,2}, \Phi_{2,2}$.

We can now calculate the quadrupole moments of 
the different states $(\lambda, I)$. These are given by

$$
\begin{aligned}
& \mathrm{Q}_{2}(\lambda, I)=\sqrt{\frac{16}{5}}_{\mathrm{g}}\left\langle\lambda, I, M=I\left|Q_{0}^{\mathrm{lab}}\right| \lambda, I, M=I\right\rangle_{\mathrm{g}} \\
& \quad=\sqrt{\frac{16}{5} \pi}\left(\frac{1-2 I}{I+1}\right) \\
& \quad \times\left\langle\Phi_{\lambda, I}(\lambda, \theta, \phi)\left|Q_{\mathrm{gg}}^{\mathrm{intr}}(\gamma, \theta, \phi)\right| \Phi_{\lambda, I}(\gamma, \theta, \phi)\right\rangle,
\end{aligned}
$$

and are easily evaluated exploiting the orthogonality properties of the $\Phi$ functions. In particular, since the product $\Phi_{1,2} \Phi_{\lambda, I}$ is always orthogonal to $\Phi_{\lambda, I}[6]$ no contribution to the quadrupole moment arises from the $\Phi_{1,2}$-term in (12), which originates from the $s^{\dagger} d+d^{\dagger} \tilde{s}$ term in the quadrupole operator $(1)$. We have therefore simply

$$
\begin{aligned}
& Q_{2}(\lambda, I)=\frac{16}{5} \pi \sqrt{\pi / 7}\left(\frac{2 I-1}{I+1}\right) N \chi \\
& \quad \times\left\langle\Phi_{\lambda, I}(\gamma, \theta, \phi)\left|\Phi_{2,2}(\gamma, \theta, \phi)\right| \Phi_{\lambda, I}(\gamma, \theta, \phi)\right\rangle .
\end{aligned}
$$

As far as the low-lying members of the ground band is concerned, one can make use of the explicit relations

$$
\begin{aligned}
& \Phi_{2,2}(\gamma, \theta, \phi) \Phi_{1,2}(\gamma, \theta, \phi) \\
& \quad=(1 / 4 \pi)\left[\frac{1}{7} \sqrt{10} \Phi_{1,2}(\gamma, \theta, \phi)\right. \\
& \left.\quad+(1 / \sqrt{6}) \Phi_{3,0}(\gamma, \theta, \phi)-\frac{1}{7} \sqrt{11} \Phi_{3,4}(\gamma, \theta, \phi)\right] \\
& \Phi_{2,2}(\gamma, \theta, \phi) \Phi_{2,2}(\gamma, \theta, \phi) \\
& \quad=(1 / 8 \pi)\left[\sqrt{2} \Phi_{0,0}(\gamma, \theta, \phi)\right. \\
& \quad-\frac{2}{21} \sqrt{10} \Phi_{2,2}(\gamma, \theta, \phi)-\frac{8}{21} \sqrt{5} \Phi_{4,2}(\gamma, \theta, \phi) \\
& \left.\quad+\frac{2}{21} \sqrt{130} \Phi_{4,4}(\gamma, \theta, \phi)+\frac{8}{21} \sqrt{2} \Phi_{2,4}(\gamma, \theta, \phi)\right]
\end{aligned}
$$

and

$$
\begin{aligned}
& \Phi_{2,2}(\gamma, \theta, \phi) \Phi_{2,4}(\gamma, \theta, \phi) \\
& \quad=(1 / \pi)\left[\frac{1}{21} \sqrt{2} \Phi_{2,2}(\gamma, \theta, \phi)-\frac{5}{84} \Phi_{4,2}(\gamma, \theta, \phi)\right. \\
& \quad+\frac{5}{63} \sqrt{\frac{5}{2}} \Phi_{2,4}(\gamma, \theta, \phi)-\frac{5}{693} \sqrt{26} \Phi_{4,4}(\gamma, \theta, \phi) \\
& \left.\quad-\frac{5}{44} \sqrt{\frac{5}{6}} \Phi_{4,6}(\gamma, \theta, \phi)\right] .
\end{aligned}
$$

With these expressions it is straightforward to calculate the corresponding quadrupole moments;

$$
\begin{aligned}
& Q_{2}\left(2_{1[\lambda=1, I=2]}^{+}\right)=\frac{4}{7} \mathrm{~N} \chi \sqrt{\frac{2}{35} \pi}, \\
& Q_{2}\left(2_{2[\lambda=2, I=2]}^{+}\right)=-\frac{4}{21} \mathrm{~N} \chi \sqrt{\frac{2}{35} \pi},
\end{aligned}
$$

and

$Q_{2}\left(4_{1[\lambda=2, I=4]}^{+}\right)=\frac{8}{9} N \chi \sqrt{\frac{2}{35}} \pi$.

These results coincide, in the limit of large values of $N$, with those given in ref. [4].

Similar arguments apply to $B(E 2)$ transitions. In general

$$
\begin{aligned}
& B\left(\mathrm{E} 2 ;\left(\lambda_{1} I_{1}\right)_{\mathrm{g}} \rightarrow\left(\lambda_{2} I_{2}\right)_{\mathrm{g}}\right) \\
& \quad=\left.\left.\frac{1}{2 I_{1}+1}\right|_{\mathrm{g}}\left\langle\lambda_{1} I_{1}\left\|\hat{Q}^{\mathrm{lab}}\right\| \lambda_{2} I_{2}\right\rangle_{\mathrm{g}}\right|^{2} \\
& \quad=\frac{1}{2 I_{1}+1}\left(\begin{array}{rrr}
I_{1} & 2 & I_{2} \\
0 & 0 & 0
\end{array}\right)^{-2} \\
& \times\left|\left\langle\Phi_{\lambda_{1} I_{1}}(\gamma, \theta, \phi)\left|Q_{\mathrm{gg}}^{\text {intr }}(\gamma, \theta, \phi)\right| \Phi_{\lambda_{2} I_{2}}(\gamma, \theta, \phi)\right\rangle\right|^{2} .
\end{aligned}
$$

Then the problem of evaluating $B(\mathrm{E} 2)$ values is again reduced to the problem of exploiting the orthogonality and recursion properties of the $\Phi$ functions. In particular one directly sees that both $\Delta \lambda= \pm 1$ and $\Delta \lambda=0, \pm 2$ are allowed, through the first and second terms in the intrinsic quadrupole moment (12). As a particular case the form (12) yields the selection rule that from the ground state only transitions to the first and second $2^{+}$states are allowed. For these transitions

$B\left(\mathrm{E} 2 ; 2_{1}^{+} \rightarrow 0_{1}^{+}\right)=\frac{1}{5} N^{2}$,

$B\left(\mathrm{E} 2 ; 22_{2}^{+} \rightarrow 0_{1}^{+}\right)=\frac{1}{70} N^{2} \chi^{2}$,

while for the transition from the second to the first $2^{+}$state one obtains

$B\left(\mathrm{E} 2 ; 2_{2}^{+} \rightarrow 2_{1}^{+}\right)=\frac{2}{7} N^{2}$.

As in the case of the quadrupole moments, these values coincide, to higher order in $N$, with the $\mathrm{O}(6)$ values obtained directly in the laboratory frame.

In a similar way one can evaluate interband transitions. In the $O(6)$ limit the first excited band (corresponding in the geometrical picture to a vibration in the $\beta$ degree of freedom around the equilibrium value $\beta_{0}$ ) can be viewed as originating from an intrinsic excited state of the form 


$$
\left|\Psi_{\beta}\right\rangle=\frac{1}{\sqrt{(N-1) !}}\left(\gamma^{\dagger}\right)^{N-1}\left(\tilde{\gamma}^{\dagger}\right)|0\rangle,
$$

where one of the basic bosons of the condensate has been promoted to the orthogonal combination

$$
\begin{aligned}
\tilde{\gamma}^{\dagger} & =(1 / \sqrt{2})\left[-s^{\dagger}+\cos \gamma d_{0}^{\dagger}\right. \\
& \left.+(1 / \sqrt{2}) \sin \gamma\left(d_{2}^{\dagger}+d_{-2}^{\dagger}\right)\right] .
\end{aligned}
$$

In analogy with (9), each member $|\lambda, I, M\rangle_{\beta}$ of the $\beta$-band is assumed to be characterized by the wave function

$$
|\lambda, I, M\rangle_{\beta}=\Phi_{\lambda, I, M}\left(\gamma, \theta_{\mathrm{i}}\right)\left|\Psi_{\beta}\right\rangle .
$$

All interband transitions between the ground- and $\beta$ bands are connected to an off-diagonal matrix element of the quadrupole operator between the two intrinsic states (2) and (26). We get in this case

$$
\begin{aligned}
B & \left(\mathrm{E} 2 ;\left(\lambda_{1} I_{1}\right)_{\beta} \rightarrow\left(\lambda_{2} I_{2}\right)_{\mathrm{g}}\right) \\
& =\left.\left.\frac{1}{2 I_{1}+1}\right|_{\beta}\left\langle\lambda_{1} I_{1}\left\|\hat{Q}^{\mathrm{lab}}\right\|^{\mathrm{lab}} \| \lambda_{2} I_{2}\right\rangle_{\mathrm{g}}\right|^{2} \\
& =\frac{1}{2 I_{1}+1}\left(\begin{array}{ccc}
I_{1} & 2 & I_{2} \\
0 & 0 & 0
\end{array}\right)^{-2} \\
& \times\left|\left\langle\Phi_{\lambda_{1} I_{1}}(\gamma, \theta, \phi)\left|Q_{\mathrm{g} \beta}^{\mathrm{intr}}(\gamma, \theta, \phi)\right| \Phi_{\lambda_{2} I_{2}}(\gamma, \theta, \phi)\right\rangle\right|^{2},
\end{aligned}
$$

where we have defined

$$
\begin{aligned}
& Q_{\mathrm{g} \beta}^{\mathrm{intr}}(\gamma, \theta, \phi)=\left\langle\Psi_{\beta}\left|\hat{Q}_{0}^{\mathrm{lab}}\right| \Psi_{\mathrm{g}}\right\rangle \\
& \quad=\left\langle\Psi_{\beta}\left|\sum_{\mu} \sqrt{\frac{4}{5} \pi}(-1)^{\mu} \hat{Q}_{\mu} Y_{2, \mu}(\theta, \phi)\right| \Psi_{\mathrm{g}}\right\rangle .
\end{aligned}
$$

The evaluation of this matrix element yields

$Q_{\mathrm{g} \beta}^{\mathrm{intr}}(\gamma, \theta, \phi)=-\frac{4 \pi \chi \sqrt{N}}{\sqrt{35}} \Phi_{2,2}(\gamma, \theta, \phi)$.

Note, in comparison with (12), the precise cancellation (occurring only in the $O(6)$ limit, i.e. for $\beta=1$ ) of the term associated with $\Phi_{1,2}(\gamma, \theta, \phi)$. The evaluation of interband transition rates are therefore once more determined by the orthogonality properties of the $\Phi$ functions. As a direct consequence of (30), there is no direct connection between $0_{\mathrm{g}}^{+}$and the first $2_{\beta}^{+}$or for any other $\Delta \lambda= \pm 1$ transition. One has instead non-vanishing transitions (corresponding to $\Delta \lambda= \pm 2$ ) from the second $2{ }_{\beta}^{+}$state to the ground state

$$
B\left(\mathrm{E} 2 ; 2_{2, \beta}^{+} \rightarrow 0_{1, \mathrm{~g}}^{+}\right)=\frac{1}{70} N \chi^{2},
$$

from the band-head of the $\beta$-band to the second $2_{\mathrm{g}}^{+}$ state of the ground band

$B\left(\mathrm{E} 2 ; 0_{1, \beta}^{+} \rightarrow 22_{2, \mathrm{~g}}^{+}\right)=\frac{1}{14} N \chi^{2}$,

and, for instance, among the first $2^{+}$states in the $\beta$ and ground band $(\Delta \lambda=0)$

$B\left(\mathrm{E} 2 ; 2_{1, \beta}^{+} \rightarrow 2_{1, \mathrm{~g}}^{+}\right)=\frac{1}{49} N \chi^{2}$.

In all cases these results correspond in leading order with those obtained in the laboratory. It should also be possible to evaluate in-band transitions rates and quadrupole moments within the $\beta$-band by evaluating the corresponding intrinsic quadrupole moment $Q_{\beta \beta}^{\text {intr }}$ between states of the form (26).

We have made use of the concept of intrinsic state for $\gamma$-unstable systems and of the associated projecting kernels in order to derive electromagnetic transition rates and quadrupole moments of the $\mathrm{O}(6)$ limit of the interacting boson model. We have to mention that attempts have been recently made [7] in order to relate the $O(6)$ dynamical symmetry to the one arising from a triaxial $(\gamma=30)$ rotor. Part of the motivation may have originated from the fact that, at first glance, to project a (asymmetric) rotor implies a more standard technique than the $\gamma$-unstable case. As shown in this letter, dealing with $\gamma$-dependent quantities in the intrinsic frame and with the $\gamma$-dependent kernels does not imply technical problems qualitatively different from those associated with the projection of a triaxial rotor with a definite value of $\gamma$.

This work was supported in part by a common grant from the Comision Asesora de Investigacion Cientifica y Tecnica (Spain) and INFN (Italy) and Project No. PB86-318.

\section{References}

[1] L. Wilets and M. Jean, Phys. Rev. C 102 (1956) 788.

[2] A. Arima and F. Iachello, Ann. Phys. (NY) 123 (1979) 468.

[3] J. Meyer-ter-Vehn, Phys. Lett. B 84 (1979) 10.

[4] P. van Isacker, Nucl. Phys. A 465 (1987) 497. 
[5] J.N. Ginocchio and M.W. Kirson, Nucl. Phys. A 350 (1980)3; Phys. Rev. Lett. 44 (1980) 1744;

A.E.L. Dieperink, O. Scholten and F. Iachello, Phys. Rev. Lett. 44 (1980) 1747;

A.E.L. Dieperink and O. Scholten, Nucl. Phys. A 346 (1980) 125 ;

J.Q. Chen and P. Van Isacker, in: Interacting Bose-Fermi systems in nuclei, ed. F. Iachello (Plenum, New York, 1981) p. 193;
J. Dukelsky, G.G. Dussel, R.P.J. Perazzo, S.L. Reich and H.M. Sofia, Nucl. Phys. A 425 (1984) 93;

A. Leviatan, in: Nuclear structure, reactions and symmetries, eds. R.A. Meyer and V. Paar, Vol. II, (World Scientific, Singapore, 1986) p. 1030.

[6] D. Bes, Nucl. Phys. 10 (1959) 373.

[7] T. Otsuka and M. Sugita, Phys. Rev. Lett. 59 (1987) 1541. 